\title{
Effect of Implementing the New Diagnostic Criteria for Noninvasive Follicular Thyroid Neoplasm with Papillary-Like Nuclear Features in King Abdulaziz University Hospital
}

\author{
Fatimah A. Alturkistani ${ }^{1,2}$ MBBS and Murad A. Alturkustani ${ }^{1}$, FRCPC, MBBS \\ 'Department of Pathology, Faculty of Medicine, \\ King Abdulaziz University and ${ }^{2}$ King Abdulaziz Medical City \\ Jeddah, Saudi Arabia
}

\section{Correspondence}

Dr. Murad A. Alturkustani

P.O. Box 80205, Jeddah 21589, Saudi Arabia

e.M: alturkustani.murad@gmail.com

Submission: 6 Jun. 2016

Accepted: $\quad 28$ Jun. 2016

\section{Citation}

Alturkistani FA, Alturkustani MA. Effect of implementing the new diagnostic criteria for noninvasive follicular thyroid neoplasm with papillary-like nuclear features in King Abdulaziz University Hospital. JKAU Med Sci 2016; 23 (3):

31-37. DOI: 10.4197/Med. 23.3.4

\begin{abstract}
Pathological diagnosis of follicular variant of papillary thyroid carcinoma has high inter and intra-observer variability among expert pathologists, affecting prognosis and management of the disease. A recent study applying strict diagnostic criteria and long-term follow up confirmed the indolent behavior of a subtype of these tumors. They recommended a nomenclature change to noninvasive follicular thyroid neoplasm with papillary-like nuclearfeatures, reflecting thelow risk of adverse outcomes. We searched the pathology archives of King Abdulaziz University Hospital from 2002-2016 for all cases diagnosed with"follicular variant of papillary thyroid carcinoma". Clinical data, and imaging findings were retrospectively reviewed. Available pathology slides were reviewed using the proposed inclusion and exclusion diagnostic criteria. We confirmed the diagnostic reproducibility of the suggested criteria. Ten out of 37 cases met the diagnostic criteria. Eight additional patients could have had their diagnosis modified if sufficient tissue samples were available. Follow up data confirmed the indolent behavior in these cases with no recurrence or adverse outcome. We concluded that application of the new diagnostic criteria for this subtype is reasonable and has major ramifications for the diagnosis and management as this will spare unnecessary thyroidectomies, radioactive iodine therapy, and their complications.
\end{abstract}

\section{Keywords}

Thyroid cancer; Noninvasive follicular thyroid neoplasm with papillary-like nuclear features 


\section{Introduction}

Multiple neoplasms of the thyroid gland exist, each with different morphological features, prognosis, and management. Well-differentiated thyroid carcinomas fall into two main categories: Papillary thyroid cancer and follicular thyroid carcinoma. Diagnosis of each type depends on certain well-defined histopathological features. Papillary thyroid cancer is the most common type with a reported increase in incidence in the last decade ${ }^{[1]}$. Different variants of papillary thyroid cancer exist with the follicular variant being most commonly diagnosed after the classical type ${ }^{[1]}$. The management of this variant was previously through total thyroidectomy, and sometimes followed by radioactive iodine ablation. Recently, 24 endocrine pathologists from 4 different continents conducted a large, retrospective study regarding this entity. They reviewed cases of the follicular variant of papillary thyroid carcinoma (FVPTC) from different institutes and applied strict inclusion and exclusion criteria. The selected cases were followed over a period of 1026 years in an attempt to define their prognosis. The study confirmed the benign behavior of a subtype of this neoplasm and recommended a change in its nomenclature to one that reflects its benign, indolent behavior: "Noninvasive follicular thyroid neoplasm with papillary-like nuclear features" (NIFTP) ${ }^{[2]}$. In our study, we applied the recommended inclusion and exclusion criteria to cases from the archives of a tertiary care center in Saudi Arabia. Our aims were to determine the applicability of these pathological criteria, and to confirm the benign behavior of these neoplasms in a different population.

\section{Methods}

Approval from the Research Committee of the Biomedical Ethics Unit, Faculty of Medicine, King Abdulaziz University was obtained prior to commencement of the study. We searched the archives of the pathology department covering the period 2002-2016 for all cases designated as "follicular variant of papillary thyroid carcinoma". We retrieved 37 cases. The clinical data (sex, age at onset and surgical intervention), imaging study, and pathological findings were retrospectively reviewed. All patients were followed up until May 2016. The available pathological slides were reviewed and the diagnostic inclusion and exclusion criteria were adopted from the recently published article ${ }^{[2]}$. The diagnostic criteria were as follows: Encapsulated or clearly demarcated tumor with a follicular growth pattern and nuclear features of papillary thyroid carcinoma (PTC) (score 2 or 3 ). The nuclear features were scored as present (1) or absent (0) for the following characteristics: Size and shape (enlargement of the nuclei with overlapping, crowding and/or elongation); nuclear membrane irregularity (irregular nuclear contour, nuclear pseudoinclusion and grooves); chromatin characteristics (nuclear clearing with margination and glassy nuclei) (Fig. 1A, 1B). The exclusion criteria were: Papillae formation in more than $1 \%$ of the tumor, the presence of psammoma bodies, an infiltrative border (Fig. 1C, 1D), and tumor necrosis, the presence of at least 3 mitoses per 10 highpower fields, and cell /morphologic characteristics of other variants of PTC (Fig. 1E, 1F).

\section{Results}

We reviewed the 37 cases found in our archives. This included 34 female and 3 male patients. The age of patients ranged from 19 to 78 years at the time of diagnosis (median age, 37 years and mean age, 40 years. The tumor size ranged from $0.3 \mathrm{~cm}$ to $2.5 \mathrm{~cm}$. Twentysix cases were excluded from the study after reviewing the available pathological slides and the reasons for exclusion are summarized in Table 1. Eleven cases were found to meet the diagnostic criteria for the new entity; one case had no follow up data and was subsequently excluded. The final number of cases included in this study was 10. A summary of the selected cases and their clinical data as well as follow up results are shown in Table 2.

We found that application of the proposed diagnostic and exclusion criteria was simple, reasonable, and reproducible. A few cases were challenging and this was mainly related to the amount of tissue sampled from the neoplasm. The proposed criteria for adequate sampling of these neoplasms were not specified; generally, assessment of the whole capsule is preferred. However, due to the well-known variation in the amount of sampling between pathologists, 8 cases in our small cohort were excluded as the sampling was considered inadequate for the diagnosis of this variant. Another major problem was dealing with multiple nodules with FVPTC features. In this scenario, only representative sections, and not the whole thyroid, were submitted for histological diagnosis. One case was associated with nodal metastasis.

Eight cases were managed initially by total thyroidectomy, one with initial lobectomy followed subsequently by complete thyroidectomy and 
Effect of Implementing the New Diagnostic Criteria for Noninvasive Follicular Thyroid Neoplasm...

F.A. Alturkistani and M. A. Alturkustani
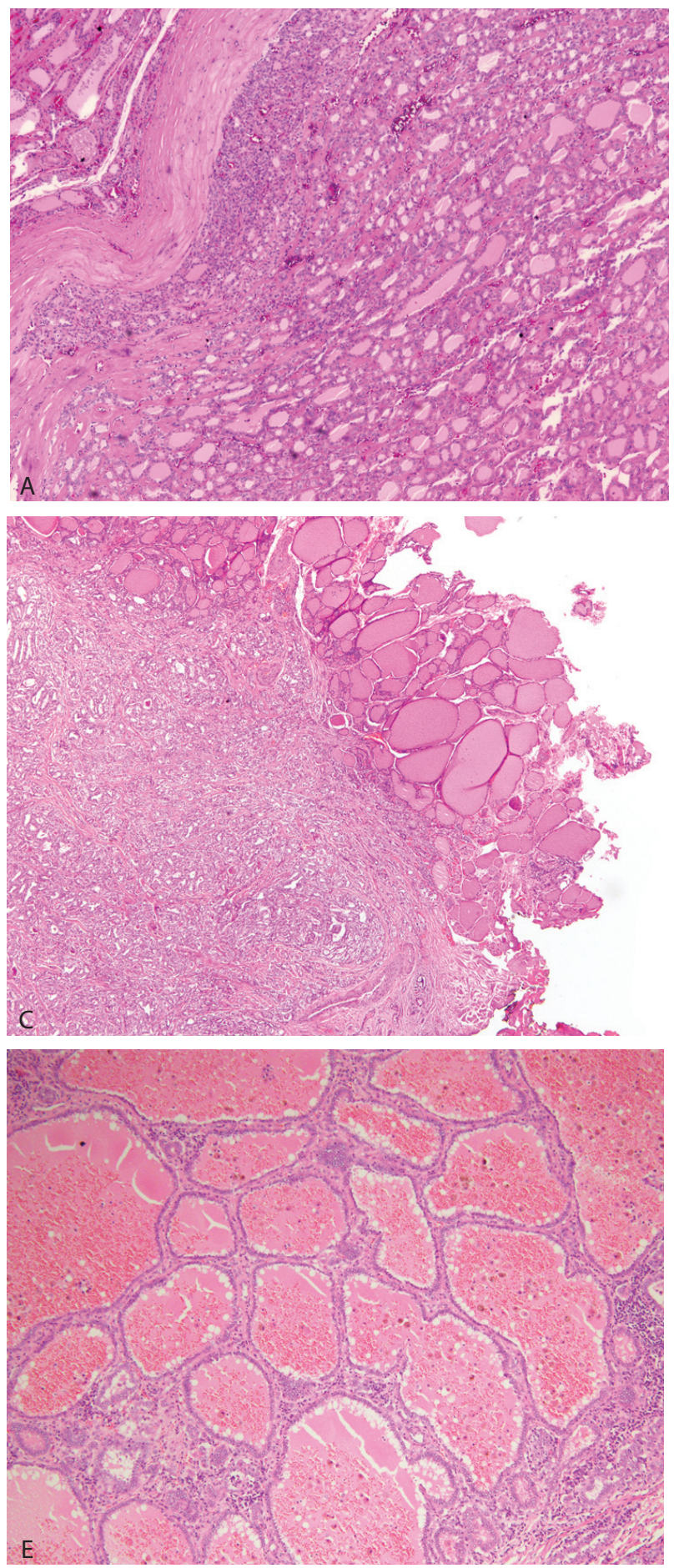
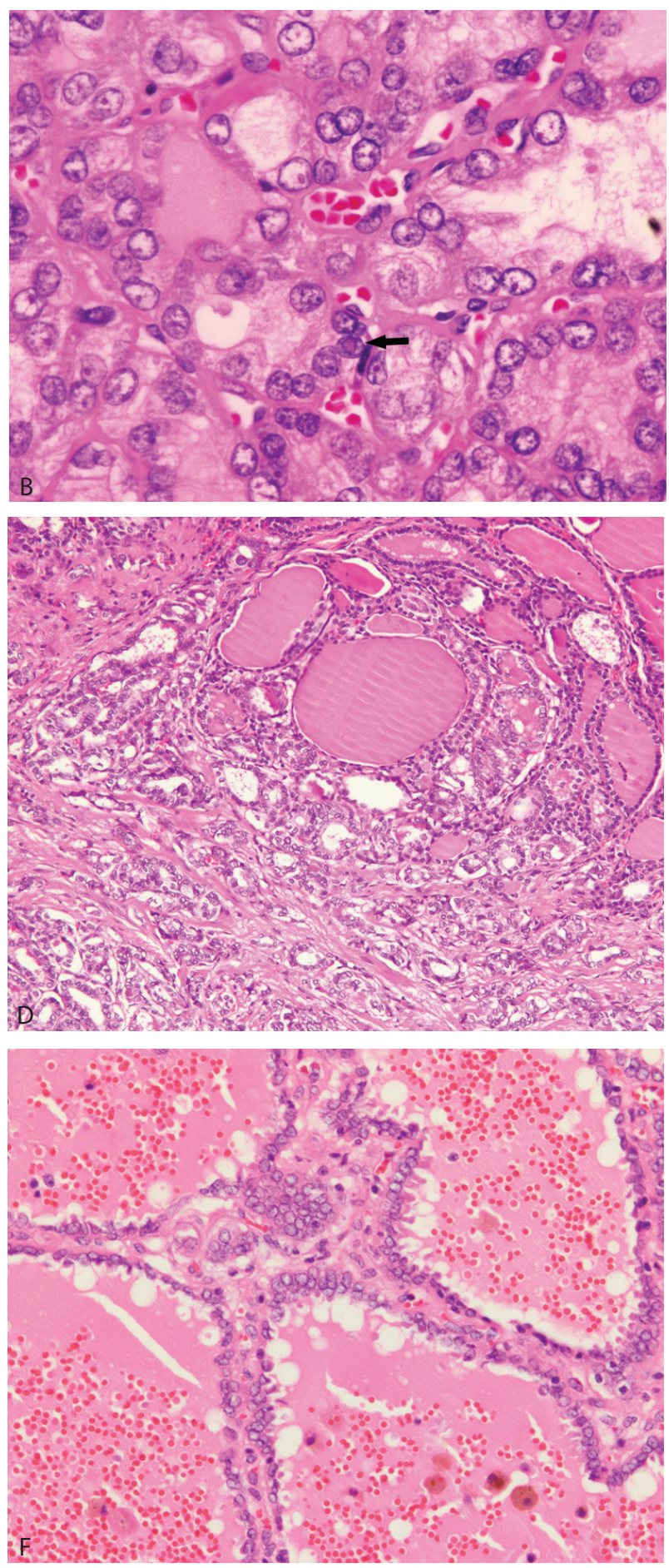

Figure 1. Classic example of NIFTP and features resulting in exclusion. A: The neoplastic cells are arranged in small follicles and the neoplasm is encapsulated by a fibrous capsule (Hematoxylin \& Eosin; original magnification: 40 x). B: Higher magnification of the neoplastic cells in (A) highlights the nuclear features of PTC. The nuclei are large, pleomorphic, with an irregular nuclear membrane, vesicular chromatin, and one nucleus shows pseudo inclusion (arrow). The nuclear score for this neoplasm is 3 (Hematoxylin \& Eosin; original magnification: $400 \mathrm{x}$ ). C: Example of an excluded case due to the infiltrative border of this non-encapsulated FVPTC (Hematoxylin \& Eosin; original magnification: 40 x). D: Higher magnification of (C) to highlight the focal infiltrative area in this neoplasm (Hematoxylin \& Eosin; original magnification: $200 \mathrm{x}$ ). E: Example of another excluded case as the follicular growth in this neoplasm is larger and should be considered as a macrofollicular variant of PTC (Hematoxylin \& Eosin; original magnification: 200 x). F: Higher magnification of (E) to show the nuclear features of PTC in the nuclei of large follicles (Hematoxylin \& Eosin; original magnification: $400 \mathrm{x}$ ). 
Table 1. Excluded cases and reasons for exclusion.

\begin{tabular}{|c|l|c|}
\hline \multicolumn{1}{|c|}{ Reason for Exclusion } & $\begin{array}{c}\text { Number of Excluded } \\
\text { Cases }\end{array}$ \\
\hline 1 & Insufficient pathological information & 8 \\
\hline 2 & Absence of follow up data & 1 \\
\hline 3 & Vascular invasion & 1 \\
\hline 4 & Capsular invasion & 3 \\
\hline 5 & Psammoma bodies & 2 \\
\hline 6 & Macrofollicular variant & 1 \\
\hline 7 & Infiltrative pattern & 3 \\
\hline 8 & Did not meet the proposed diagnostic criteria for the nuclear features & 1 \\
\hline 9 & $>1 \%$ papillary architecture & 2 \\
\hline 10 & Multiple nodules in background of Hashimoto's & 2 \\
\hline 11 & Multiple nodules some with conventional PTC & 3 \\
\hline \multicolumn{2}{r}{ Total number } & $\mathbf{2 7}$ \\
\hline
\end{tabular}

Table 2. Summary of the clinical features and follow up data of the included cases.

\begin{tabular}{|c|c|l|l|l|c|}
\hline N. & $\begin{array}{c}\text { Age at the Time of } \\
\text { Diagnosis }\end{array}$ & Gender & Tumor size & \multicolumn{1}{|c|}{ Management } & $\begin{array}{c}\text { Follow up } \\
\text { Duration* }\end{array}$ \\
\hline 1 & $41 \mathrm{y}$ & Female & $2.5 \mathrm{~cm}$ & Total thyroidectomy & 4 months \\
\hline 2 & $49 \mathrm{y}$ & Female & $0.4 \mathrm{~cm}$ & $\begin{array}{l}\text { Lobectomy and isthmectomy followed by completion } \\
\text { thyroidectomy and radioactive iodine therapy }\end{array}$ & 18 months \\
\hline 3 & $38 \mathrm{y}$ & Female & $0.4 \mathrm{~cm}$ & Total thyroidectomy & 6 months \\
\hline 4 & $50 \mathrm{y}$ & Female & $0.5 \mathrm{~cm}$ & Total thyroidectomy & 4 years \\
\hline 5 & $37 \mathrm{y}$ & Female & $1.2 \mathrm{~cm}$ & Total thyroidectomy & 4 years \\
\hline 6 & $36 \mathrm{y}$ & Female & $0.5 \mathrm{~cm}$ & Total thyroidectomy & 4 years \\
\hline 7 & $53 \mathrm{y}$ & Female & $0.3 \mathrm{~cm}$ & Total thyroidectomy & 6 years \\
\hline 8 & $35 \mathrm{y}$ & Female & $0.3 \mathrm{~cm}$ & Total thyroidectomy & 5 years \\
\hline 9 & $38 \mathrm{y}$ & Female & $1.5 \mathrm{~cm}$ & Total thyroidectomy & 2 years \\
\hline 10 & $19 \mathrm{y}$ & Female & $1.2 \mathrm{~cm}$ & Lobectomy & \\
\hline
\end{tabular}

All patients were free of the disease with no evidence of recurrence or metastasis at the last follow up visit.

radioactive iodine and one by lobectomy alone. All cases were followed up until May 2016 and none showed adverse events including metastasis or recurrence. This data supports the benign, indolent behavior of this thyroid neoplastic entity.

\section{Discussion}

Well-differentiated thyroid cancer includes 2 variants: Follicular carcinoma (FC) and papillary thyroid carcinoma ${ }^{[1]}$. The diagnosis of follicular carcinoma depends on detection of capsular and or vascular invasion ${ }^{[1]}$, while the diagnosis of papillary carcinoma depends on the presence of papillary formation and certain nuclear features (namely PTC-type nuclei) even in the absence of capsular and or vascular invasion ${ }^{[3]}$. The nuclear features include optically clear nuclei, nuclear grooves, overlapping, pseudoinclusions, enlargement, and irregularities of the nuclear contours.

The FVPTC is characterized by follicles lined by cells with PTC-type nuclei but with no papillae formation. The name of this entity was introduced by Lindsay in $1960^{[4]}$ and further delineated by Chen and Rosai in $1977^{[5]}$. As it is a variant of PTC, nuclear features alone have been considered sufficient to label this variant as malignant ${ }^{[5]}$ even in the absence of capsular and or vascular invasion. The pathological diagnosis of this variant has suffered from high inter-observer ${ }^{[6-8]}$ and even intra-observer variability among experts in thyroid pathology ${ }^{[8]}$. Additionally, pathologists from many countries use different thresholds, for example an American pathologist diagnosed PTC at a higher frequency compared to a Japanese pathologist ${ }^{[6]}$. As such, there has been controversy in the literature 
regarding diagnosis, treatment, and the behavior of this entity.

The major reason for the controversy regarding the pathological diagnosis was that different expert pathologists have different thresholds for accepting what nuclear features were sufficient in a follicular lesion to call it FVPTC. This resulted in three possibilities for a follicular lesion with questionable PTC-nuclear features: First, the nuclear features were considered sufficient and subsequently termed FVPTC and considered malignant. Second, the nuclear features were not sufficient and subsequently the lesions were considered follicular neoplasms and if they demonstrated capsular and/or vascular invasion, they were considered malignant. The third was to accept the limitations and name these nuclear features as equivocal. The latter situation was approached by William, on behalf of the Chernobyl Pathologists Group, who proposed a different terminology to deal with these $\operatorname{cases}^{[9]}$. The proposal was to admit to our limitations and the uncertainty of this group and to look for other features of malignancy (namely capsular and/or vascular invasion). If these were present, then the neoplasm would be called a well-differentiated carcinoma not otherwise specified (WDC-NOS). While if they were absent, then the neoplasm would be termed a well-differentiated tumor of uncertain malignant potential (WDT-UMP) ${ }^{[9]}$. For the latter group, owing to their extremely good prognosis, they suggested that no further treatment is required. However, this proposition was not widely accepted among other experts in the field ${ }^{[1]}$.

The importance of concentrating on the capsule in FVPTC and to classify these tumors into encapsulated (invasive and non-invasive) and non-encapsulated was clearly demonstrated by Liu et al. ${ }^{[10]}$. In this study, 43 non-invasive encapsulated tumors had no evidence of recurrence or lymph node metastasis with a median follow-up of 10.8 years in contrast to the other groups (namely invasive encapsulated and the infiltrative/ diffuse non-encapsulated), which behaved like a classic papillary thyroid carcinoma ${ }^{[10]}$. In another approach to confirm the favorable prognosis of this entity, Piana et al. ${ }^{[11]}$ reviewed the follow up data of 1009 cases of thyroid carcinoma for an average of 11.9 years or until death. They selected 67 fatal cases and reviewed the pathological diagnosis. None of these cases were encapsulated FVPTC although there were 66 cases of FVPTC in the initial cohort ${ }^{[11]}$. However, this study was limited, as it did not distinguish between invasive and non-invasive cases.
Although FVPTC with capsular and/or vascular invasion behaves as a malignant neoplasm, the behavior of the remaining cases was unclear. One group should be termed "cancer" as they behave like conventional PTC, while the other behaves in a benign fashion. Due to the uncertain behavior of FVPTC with no capsular and/or vascular invasion, different treatments have been offered to patients. Institutions with an excellent follow up system and compliant patients may offer lobectomy with frequent follow up assessment, while others may decide to treat with these patients by complete thyroidectomy with or without radioactive iodine therapy. However, as a result of the recent study by Nikiforov et al. ${ }^{[2]}$ and the consensus change by experts in the field, these treatments may change.

With major advances in the diagnosis, it is clear that certain features apart from the presence of PTC-type nuclei are extremely important for these neoplasms and their indolent behavior. In order to maintain the classification of this variant and its indolent behavior, the diagnostic and exclusion criteria must be strictly applied. In the current study, we found one of the major variables that affected diagnosis was the sampling adequacy. There is variability in what is accepted as "adequate" sampling between institutes and pathologists, especially when sampling thyroid neoplasms. This variation could be anywhere from the accepted general sampling rule for any neoplasm (at least 1 section $/ 1 \mathrm{~cm}$ ) to sampling the whole thyroid. Although there is no minimum accepted adequate sampling for this diagnosis, assessment of the whole capsule is preferable for diagnosis of this variant. We excluded 8 cases from our small cohort as we considered them to be inadequately sampled to diagnose this specific variant. Another important issue was how to address multifocal NIFTP neoplasms. In our cohort, we found one case of multifocal NIFTP with nodal metastasis. Although the nodules that were sampled were done so adequately, not all the nodules were sampled and as such some of the smaller unsampled nodules may have had features of conventional PTC rather than NIFTP. Therefore, in patients with multifocal neoplasms, we recommend that either all nodules or the whole thyroid are sampled for the diagnosis of NIFTP (multifocal).

As this is the first entity to be converted from "cancer' to neoplasm with benign behavior, it will have a huge impact on patients, their insurance, society, as well as the health care budget. It will also greatly influence our tertiary care center as 10 out 37 cases (27\%) from our pathology archive will have to undergo 
a nomenclature change from the word "cancer" to "neoplasm". This number would have been higher if adequate sampling and good follow up data were available in all cases. These changes affect both the pathologist and treating clinician. For the pathologist, we cannot over-emphasize the importance of adequate sampling of thyroid neoplasms in order to diagnose this variant. As this is a newly introduced variant, clinicians who read the pathology report should be aware of this entity and may discuss cases with a diagnosis of FVPTC with the pathologist to include the possibility of NIFTP.

\section{Conclusion}

The application of the new well-defined diagnostic criteria for NIFTP is reasonable and reproducible. The application of these criteria to cases previously diagnosed as FVPTC may change the diagnosis from "cancer" to neoplasm with indolent behavior (NIFTP). A significant number of patients would benefit from this modification both psychologically and in terms of their management, as this will spare unnecessary treatment such as thyroidectomies, radioactive iodine therapy and their complications. The major impact of this modification to the nomenclature should be maintained by awareness of these changes and the strict application of inclusion and exclusion criteria for diagnosis.

\section{Conflict of Interest}

The authors has no conflict of interest.

\section{Disclosure}

None of the authors received any type of commercial support either in forms of compensation or financial for this study. They have no financial interest in any of the products or devices, or drugs mentioned in this article.

\section{Ethical Approval}

Obtained.

\section{References}

[1] DeLellis RA, Lloyd RV, Heitz PU, Eng C (Eds). Pathology and genetics of tumors of endocrine organs. In: World Health Organization Classification of Tumors. Lyon: IARC P, 2004.

[2] Nikiforov YE, Seethala RR, Tallini G, Baloch ZW, Basolo F, Thompson LD, Barletta JA, Wenig BM, AI Ghuzlan A, Kakudo K, Giordano TJ, Alves VA, Khanafshar E, Asa SL, El-Naggar AK, Gooding WE, Hodak SP, Lloyd RV, Maytal G, Mete O, Nikiforova MN, Nose V, Papotti M, Poller DN, Sadow PM, Tischler AS, Tuttle RM, Wall KB, LiVolsi VA, Randolph GW,
Ghossein RA. Nomenclature Revision for Encapsulated FollicularVariant of Papillary Thyroid Carcinoma: A Paradigm Shift to Reduce Overtreatment of Indolent Tumors. JAMA Oncol 2016; 2(8): 1023-1029.

[3] Rosai J. The encapsulated follicular variant of papillary thyroid carcinoma: back to the drawing board. Endocr Pathol 2010; 21(1): 7-11.

[4] Lindsay S. Carcinoma of the thyroid gland; a clinical and pathologic study of 293 patients at the University of California Hospital. In: Carcinoma of the Thyroid Gland; a Clinical and Pathologie Study of 293 Patients at the University of California Hospital. Springfield, IL: Thomas, 1960.

[5] Chen KT, Rosai J. Follicular variant of thyroid papillary carcinoma: a clinicopathologic study of six cases. Am J Surg Pathol 1977; 1(2): 123-130.

[6] Hirokawa M, Carney JA, Goellner JR, DeLellis RA, Heffess CS, Katoh R, Tsujimoto M, Kakudo K. Observer variation of encapsulated follicular lesions of the thyroid gland. Am J Surg Pathol 2002; 26(11): 1508-1514.

[7] Lloyd RV, Erickson LA, Casey MB, Lam KY, Lohse CM, Asa SL, Chan JK, DeLellis RA, Harach HR, Kakudo K, LiVolsi VA, Rosai J, Sebo TJ, Sobrinho-Simoes M, Wenig BM, Lae ME. Observer variation in the diagnosis of follicular variant of papillary thyroid carcinoma. Am J Surg Pathol 2004; 28(10): 1336-1340.

[8] Elsheikh TM, Asa SL, Chan JK, DeLellis RA, Heffess CS, LiVolsi VA, Wenig BM. Interobserver and intraobserver variation among experts in the diagnosis of thyroid follicular lesions with borderline nuclear features of papillary carcinoma. Am J Clin Pathol 2008; 130(5): 736-744.

[9] Williams ED. Guest Editorial: Two Proposals Regarding the Terminology of Thyroid Tumors. Int J Surg Pathol 2000; 8(3): 181-183.

[10] Liu J, Singh B, Tallini G, Carlson DL, Katabi N, Shaha A, Tuttle RM, Ghossein RA. Follicular variant of papillary thyroid carcinoma: a clinicopathologic study of a problematic entity. Cancer 2006; 107(6): 1255-1264.

[11] Piana S, Frasoldati A, Di Felice E, Gardini G, Tallini G, Rosai J. Encapsulated well-differentiated follicular-patterned thyroid carcinomas do not play a significant role in the fatality rates from thyroid carcinoma. Am J Surg Pathol 2010; 34(6): 868-872. 


\title{
أثز تطبيق المعاييز الجديدة لتشخيص نوع من أنواع ورم الغدة الدرقية في مستشثى الملك عبدالعزيز الجامعي
}

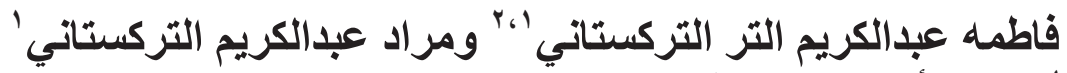

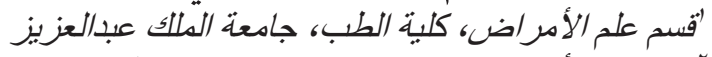

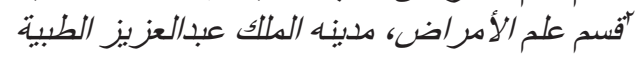 \\ جلة ـ المملكة العربية السعودية مانهي
}

المستخلص. توصلت دراسة حديثة انه من المكن تعديل تشخيص نوع من أنواع سرطان الغدة الدرقية عبر تطبيق معايير

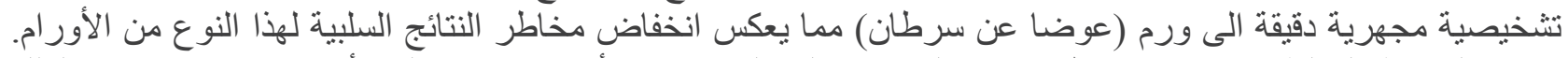

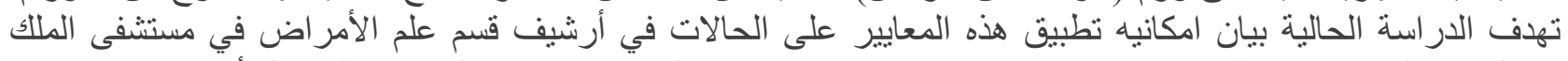

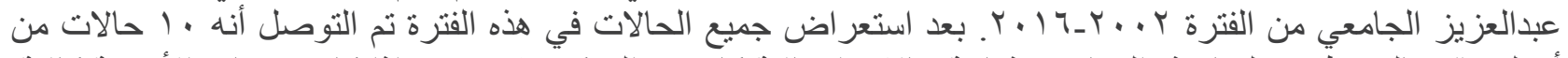

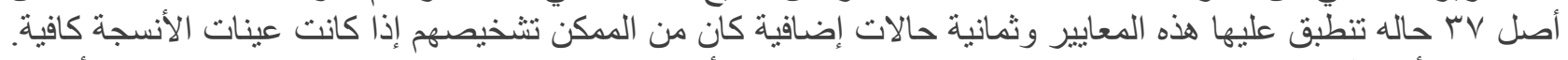

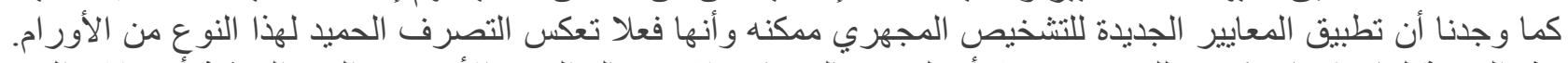

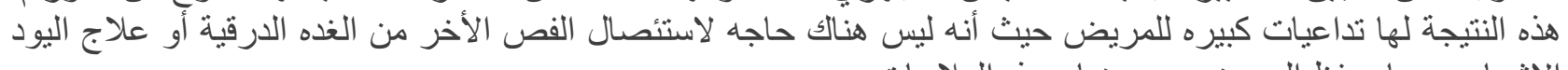
الاشعاعي مما يحفظ المريض من مضار هذه لتهات العلاجات. 